\title{
Canadian English
}

National Cancer Institute

\section{Source}

National Cancer Institute. Canadian English. NCI Thesaurus. Code C160184.

A form of the Eng lish language spoken in Canada. 